\title{
Alternative synthesis of (+/-) Cherylline dimethyl ether
}

\author{
Reshma Kurangi, Suvidha Kinalekar, Santosh Tilve* and Janardan Kirtany \\ Department of Chemistry, Goa University, Taleigao Plateau, Goa 403 206, India \\ E-mail: stilve@unigoa.ac.in
}

Dedicated to Professor S. K. Paknikar on his $73^{\text {rd }}$ birthday

\begin{abstract}
An alternative synthesis of isoquinoline alkaloid (+/-) cherylline dimethyl ether is reported. The steps involved are acid-catalyzed Michael addition of veratrole to $p$-methoxycinnamic acid, Curtius rearrangement, reduction of isocyanate intermediate followed by Pictet-Spangler cyclization.
\end{abstract}

Keywords: Michael reaction, Curtius reaction, Pictet-Spangler cyclization, tetrahydroisoquinoline

\section{Introduction}

Cherylline $\mathbf{1}$ and latifine $\mathbf{2}$ are the two 4-aryltetrahydroisoquinoline alkaloids isolated from Amaryllidaceae plants. ${ }^{1}$ Apart from the natural existence, 4-aryltetrahydroisoquinolines are of interest due to various pharmacological activities. ${ }^{2}$ For example, nomifensine ${ }^{3} \mathbf{3}$ and dichlofensine 4 exhibit central nervous system activity and inhibit serotonin and dopamine uptake mechanisms (Scheme 1).<smiles>COc1cc2c(cc1O)CN(C)CC2c1ccc(O)cc1</smiles>

1<smiles>COc1ccc2c(c1O)C(c1ccc(O)cc1)CN(C)C2</smiles>

2<smiles>CN1Cc2c(N)cccc2C(c2ccccc2)C1</smiles>

3<smiles>COc1ccc2c(c1)CN(C)CC2c1ccc(Cl)c(Cl)c1</smiles>

4

\section{Scheme 1}


There are several reports ${ }^{5}$ on the syntheses of (+/-) cherylline and of (+/-) latifine which include some efficient chiral syntheses. Most of the reported methods for the synthesis of $(+/-)$ cherylline are either multistep or low yielding. In continuation of our work ${ }^{6}$ in developing efficient methods for the synthesis of alkaloids, we report herein an alternative efficient synthesis of $(+/-)$ cherylline dimethyl ether.

\section{Results and Discussion}

Our retrosynthetic analysis of (+/-) cherylline dimethyl ether 5 is depicted in scheme 2 . We anticipated that $\mathbf{5}$ could be constructed from amine $\mathbf{6}$ via a Pictet-Spangler ring annulation which, in turn, could be obtained by reduction of the corresponding isocyanate. The required isocyanate would arise from the acid 7 via Curtius reaction. Lastly, acid 7 was to be assembled by Michael addition of veratrole to $p$-methoxycinnamic acid.

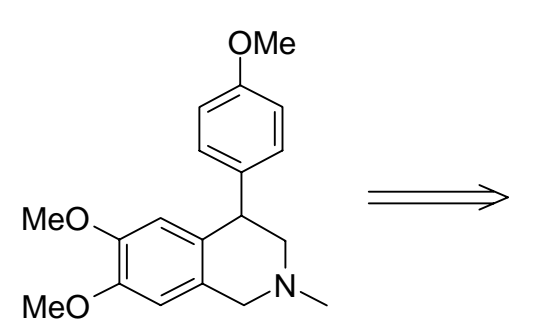

5

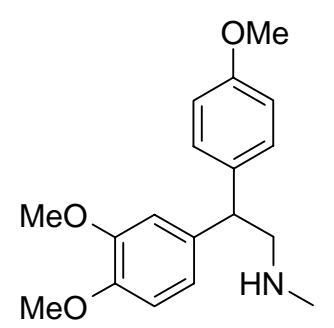

6

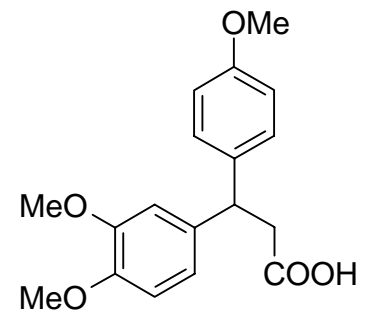

7

\section{Scheme 2}

Thus, veratrole was subjected to Michael reaction in presence of $75 \% \mathrm{H}_{2} \mathrm{SO}_{4}$ to obtain 3(3,4-dimethoxyphenyl)-3-(4-methoxyphenyl)propanoic acid ${ }^{5 \mathrm{n}} 7$ in $40 \%$ yield. Use of TFA instead of $75 \% \mathrm{H}_{2} \mathrm{SO}_{4}$ for alkylation improved the yield to $92 \%$. Modified Curtius reaction ${ }^{7}$ using diphenyl phosphorazidate (DPPA) gave low yield of the isocyanate in our hands. Resorting to the more conventional Curtius conditions using ethyl chloroformate gave isocyanate $\mathbf{9}$ in $93.7 \%$ yield. Immediate reduction of the isocyanate 9 by lithium aluminium hydride gave expected amine 6 in $82.1 \%$ yield. The crude amine 6, without further purification, on PictetSpangler reaction gave (+/-) cherylline dimethyl ether 5 in $67 \%$ yield (Scheme 3 ). The overall yield of the 4-step synthesis was found to be $47.7 \%$ from $p$-methoxycinnamic acid $\mathbf{8}$, and is better than most of the reported methods. 
<smiles>COc1ccc(/C=C/C(=O)O)cc1</smiles><smiles>CCC(C)C(=O)NCC(c1ccc(OC)cc1)c1ccc(OC)c(OC)c1</smiles>

Scheme 3. (a) Veratrole, TFA, reflux, $2.0 \mathrm{~h}, 92.0 \%$ (b)(i) $\mathrm{ClCO}_{2} \mathrm{Et}, \mathrm{N}$-methylmorpholine, acetone, $\mathrm{NaN}_{3} / \mathrm{H}_{2} \mathrm{O}, 0{ }^{\circ} \mathrm{C}$ (ii) Toluene, $110{ }^{\circ} \mathrm{C}, 1.0 \mathrm{~h}, 93.7 \%$ (c) $\mathrm{LAH}, \mathrm{THF}$, reflux, $18.0 \mathrm{~h}$, $82.5 \%$ (d) $\mathrm{HCHO}$, acetic acid, $80{ }^{\circ} \mathrm{C}, 3.0 \mathrm{~h}, 67 \%$.

In conclusion, we have devised a very short and efficient method for the synthesis of (+/-) cherylline dimethyl ether. This simple and facile nature of tetrahydroisoquinoline synthesis should allow the construction of a wide variety of interesting and useful analogous molecules.

\section{Experimental Section}

General Procedures. Melting points were determined by Thiele's tube apparatus and are uncorrected. IR spectra were recorded on Shimadzu FT-IR, IR Prestige-21 (KBr pellet or neat sample). ${ }^{1} \mathrm{H}$ NMR and ${ }^{13} \mathrm{C}$ NMR spectra were recorded on $300 \mathrm{MHz}$ Bruker instruments. The multiplicities of carbon signals were obtained from a distortionless enhancement by polarization transfer (DEPT). Coupling constants $(\mathrm{J})$ are given in $\mathrm{Hz}$ and rounded to the nearest $0.1 \mathrm{~Hz}$. The TLC plates were developed with ethyl acetate (EA)-hexanes. The silica gel used for column chromatography was Loba Chemie 60-120 mesh size. Nitrogen was used as the inert atmosphere. The glassware was equipped with a rubber septum and reagent transfer was effected by syringe techniques. Tetrahydrofuran (THF) was distilled over lithium aluminium hydride and stored over 
sodium wire. Acetone was distilled from potassium permanganate before storage over anhydrous potassium carbonate.

Preparation of 3-(3,4-dimethoxyphenyl)-3-(4-methoxyphenyl)propanoic acid (7). A solution of $p$-methoxycinnamic acid $(0.75 \mathrm{~g}, 4.21 \mathrm{mmol})$ and veratrole $(0.9 \mathrm{~g}, 6.52 \mathrm{mmol})$ in trifluoroacetic acid (TFA) $(5 \mathrm{~mL})$ was refluxed for $2 \mathrm{~h}$. TFA was distilled at atmospheric pressure and saturated $\mathrm{NaHCO}_{3}(15 \mathrm{~mL})$ was added. This aqueous phase was washed with ether (3 X $10 \mathrm{~mL})$ and acidified with conc $\mathrm{HCl}$ to give $1.47 \mathrm{~g}$ of crude 3-(3,4-dimethoxyphenyl)-3-(4methoxyphenyl)propanoic acid 7. The crude 7 was purified by column chromatography on silica gel (ethyl acetate-hexanes $1: 3)$ to yield 7 as white solid $(1.22 \mathrm{~g}, 92 \%) ; \mathrm{mp} 83{ }^{\circ} \mathrm{C}$. IR $\left(\mathrm{KBr}, \mathrm{cm}^{-1}\right)$ 3100-3300 br $(\mathrm{OH})$ and $1712(\mathrm{C}=\mathrm{O}) .{ }^{1} \mathrm{H}$ NMR $\left(300 \mathrm{MHz}, \mathrm{CDCl}_{3}\right) \delta 3.04(2 \mathrm{H}, \mathrm{d}, \mathrm{J}=7.8 \mathrm{~Hz},-\mathrm{CH}-$ $\left.\mathrm{CH}_{2^{-}}\right), 3.79\left(3 \mathrm{H}, \mathrm{s},-\mathrm{OCH}_{3}\right), 3.83\left(3 \mathrm{H}, \mathrm{s},-\mathrm{OCH}_{3}\right), 3.85\left(3 \mathrm{H}, \mathrm{s},-\mathrm{OCH}_{3}\right), 4.44(1 \mathrm{H}, \mathrm{t}, \mathrm{J}=7.8 \mathrm{~Hz}$, $\left.\mathrm{CH}-\mathrm{CH}_{2}\right), 6.73-7.27(7 \mathrm{H}, \mathrm{m}, \mathrm{ArH}) .{ }^{13} \mathrm{C} \mathrm{NMR}\left(75 \mathrm{MHz}, \mathrm{CDCl}_{3}\right) \delta 40.89\left(\mathrm{CH}_{2}\right), 45.41(\mathrm{Ar}-\mathrm{CH}-$ Ar), $55.15\left(\mathrm{OCH}_{3}\right), 55.76\left(\mathrm{OCH}_{3}\right), 55.79\left(\mathrm{OCH}_{3}\right), 111.20\left(2 \mathrm{X} \mathrm{CH}_{\mathrm{ar}}\right) 113.94\left(2 \mathrm{X} \mathrm{CH}_{\mathrm{ar}}\right), 119.17$ $\left(\mathrm{CH}_{\mathrm{ar}}\right) 128.24\left(2 \mathrm{X} \mathrm{CH}_{\mathrm{ar}}\right), 135.6\left(\mathrm{C}_{\mathrm{ar}}\right), 136.23\left(\mathrm{C}_{\mathrm{ar}}\right), 147.61\left(\mathrm{OC}_{\mathrm{ar}}\right), 148.87\left(\mathrm{OC}_{\mathrm{ar}}\right), 158.14\left(\mathrm{OC}_{\mathrm{ar}}\right)$, $177.78(\mathrm{COOH})$.

Preparation of (+/-) cherylline dimethyl ether (5). Ethyl chloroformate $(0.65 \mathrm{~mL}, 6.65 \mathrm{mmol})$ in dry acetone $(2 \mathrm{~mL})$ was added to the mixture of acid $7(2.00 \mathrm{~g}, 6.33 \mathrm{mmol})$, N-methylmorpholine $(0.80 \mathrm{~g}, 7.85 \mathrm{mmol})$ and acetone $(5 \mathrm{~mL})$ under inert atmosphere at $0{ }^{\circ} \mathrm{C}$ over $35 \mathrm{~min}$. The mixture was then stirred for $1.0 \mathrm{~h}$ at $0{ }^{\circ} \mathrm{C}$. A chilled solution of sodium azide $(0.82 \mathrm{~g}, 12.66 \mathrm{mmol})$ in 6 $\mathrm{mL} \mathrm{H}_{2} \mathrm{O}$ was added over a period of $20 \mathrm{~min}$. After stirring for $1.0 \mathrm{~h}$ at $0{ }^{\circ} \mathrm{C}$, the reaction mixture was poured into chilled water. The product was extracted in toluene $(6 \mathrm{X} 10 \mathrm{~mL})$ and combined organic extracts were dried over $\mathrm{Na}_{2} \mathrm{SO}_{4}$. The toluene layer was stirred at $110{ }^{\circ} \mathrm{C}$ for $1.0 \mathrm{~h}$. Concentration of reaction mixture under vacuum gave isocyanate 9 (1.86 g, $93.75 \%)$, which was pure (TLC). IR $\left(\mathrm{KBr}, \mathrm{cm}^{-1}\right) 2260(\mathrm{~N}=\mathrm{C}=\mathrm{O})$.

Isocyanate $9(1.86 \mathrm{~g}, 5.94 \mathrm{mmol})$ in dry THF $(5 \mathrm{~mL})$ was slowly added to the suspension of lithium aluminium hydride (LAH) $(1.2 \mathrm{~g}, 29.5 \mathrm{mmol})$ in THF $(10 \mathrm{~mL})$ under inert atmosphere. After refluxing for $18.0 \mathrm{~h}$, the reaction mixture was cooled to $5{ }^{\circ} \mathrm{C}$ and chilled water was slowly added to it. The aluminium hydroxide formed was filtered over celite and washed with boiling chloroform. The filtrate also was extracted with boiling chloroform (6 X $15 \mathrm{~mL})$. All the organic extracts and washings were combined, dried over $\mathrm{Na}_{2} \mathrm{SO}_{4}$, filtered and concentrated to obtain $1.56 \mathrm{~g}\left(82.1 \%\right.$ ) of crude 6 which was pure enough (TLC). IR (film, $\left.\mathrm{cm}^{-1}\right) 3124$ (NH).

A mixture of 6 (1.26 g, $4.18 \mathrm{mmol})$, formaldehyde $(0.44 \mathrm{~g}, 4.39 \mathrm{mmol})$ and acetic acid $(0.26$ $\mathrm{g}, 4.39 \mathrm{mmol}$ ) was stirred at $90{ }^{\circ} \mathrm{C}$ under inert atmosphere for $4.0 \mathrm{~h}$. After cooling to room temperature, the reaction mixture was basified with saturated $\mathrm{NaHCO}_{3}$ solution. This basified solution was extracted in hot chloroform ( 8 X $15 \mathrm{~mL})$, dried, filtered and concentrated to obtain $1.1 \mathrm{~g}$ of crude product. Purification of crude product by column chromatography on basic alumina using Ethyl acetate: Hexanes (15:85) as an eluent gave 5 (0.87 g, $67 \%$ ), as a white solid, mp 90-92 ${ }^{\circ} \mathrm{C}\left(\mathrm{lit}^{3 \mathrm{a}} \mathrm{mp} 87-89{ }^{\circ} \mathrm{C}\right) \mathrm{UV}(\mathrm{MeOH}, \mathrm{nm}, \varepsilon): 283$ (5665), 277.5 (5683), 234 (11008), 226 (10876). IR (KBr, cm $\left.{ }^{-1}\right): 1610,1514$. HRMS m/z calculated for $\mathrm{C}_{19} \mathrm{H}_{23} \mathrm{NO}_{3}$ - 
314.1756 [M+1], found - 314.1751. ${ }^{1} \mathrm{H}$ NMR $\left(300 \mathrm{MHz}, \mathrm{CDCl}_{3}\right): \delta 2.72\left(3 \mathrm{H}, \mathrm{s}, \mathrm{NCH}_{3}\right), 2.85$ $(1 \mathrm{H}, \mathrm{s}(\mathrm{br}), \mathrm{Ar}-\mathrm{HCH}-\mathrm{N}), 3.35(1 \mathrm{H}, \mathrm{s}(\mathrm{br}), \mathrm{Ar}-\mathrm{HCH}-\mathrm{N}), 3.65\left(3 \mathrm{H}, \mathrm{s}, \mathrm{OCH}_{3}\right), 3.82\left(3 \mathrm{H}, \mathrm{s}, \mathrm{OCH}_{3}\right)$, $3.87\left(3 \mathrm{H}, \mathrm{s}, \mathrm{OCH}_{3}\right), 3.96\left(2 \mathrm{H}, \mathrm{s}(\mathrm{br}), \mathrm{CH}-\mathrm{CH}_{2}-\mathrm{N}\right), 4.49(1 \mathrm{H}, \mathrm{s}(\mathrm{br}), \mathrm{Ar}-\mathrm{CH}-\mathrm{Ar}), 6.35(1 \mathrm{H}, \mathrm{s}, 5-$ $\mathrm{CH}), 6.58(1 \mathrm{H}, \mathrm{s}, 8-\mathrm{CH}), 6.87\left(2 \mathrm{H}, \mathrm{d}, \mathrm{J}=8.5 \mathrm{~Hz}, 2^{\prime}-\mathrm{CH}\right), 7.12\left(2 \mathrm{H}, \mathrm{d}, \mathrm{J}=8.5 \mathrm{~Hz}, 3^{\prime}-\mathrm{CH}\right) .{ }^{13} \mathrm{C}$ NMR (75 MHz, $\left.\mathrm{CDCl}_{3}\right) \delta(\mathrm{ppm}): 42.38\left(\mathrm{NCH}_{3}\right), 43.97$ (ArCHAr), $55.27\left(\mathrm{OCH}_{3}\right), 55.89\left(\mathrm{OCH}_{3}\right)$, $55.98\left(\mathrm{OCH}_{3}\right), 56.08\left(\mathrm{NCH}_{2}\right), 59.83\left(\mathrm{NCH}_{2} \mathrm{Ar}\right), 108.65\left(\mathrm{CH}_{\mathrm{ar}}\right), 111.79\left(\mathrm{CH}_{\mathrm{ar}}\right), 114.21(2 \mathrm{X}$ $\left.\mathrm{CH}_{\mathrm{ar}}\right), 127.87\left(\mathrm{C}_{\mathrm{ar}}\right), 129.29\left(\mathrm{C}_{\mathrm{ar}}\right), 129.94(2 \mathrm{X} \mathrm{C} \mathrm{ar}), 133.84\left(\mathrm{C}_{\mathrm{ar}}\right), 148.24\left(\mathrm{OC}_{\mathrm{ar}}\right), 148.59\left(\mathrm{OC}_{\mathrm{ar}}\right)$, $158.12\left(\mathrm{OC}_{\mathrm{ar}}\right)$.

\section{Acknowledgements}

We thank University Grants Commission, New Delhi (India), and Council of Scientific and Industrial Research (CSIR), New Delhi (India), for financial support. We also thank National Institute of Oceanography, Dona-Paula, Goa, and Indian Institute of Science, Bangalore, for spectral analysis.

\section{References}

1. (a) Brossi, A.; Grethe, G.; Teitel, S.; Wildman, W. C.; Bailey, D. T. J. Org. Chem. 1970, 35, 1100. (b) Kobayashi, S.; Tokumoto, T.; Taira, Z. J. Chem. Soc., Chem. Commun. 1984, 1043.

2. (a) Charifson, P. S. Drugs Fut. 1989, 14, 1179. (b) Guillon, J.; Dallemagne, P.; Leveque, H.; Duval, R.; Rault, S. Pharm Sci. 1997, 325 and the references cited therein.

3. (a) Zara-Kaczaim, E.; György, L.; Deak, G.; Seregi, A.; Doda, M. J. Med. Chem. 1986, 29, 1189. (b) Ulin, J.; Gee, A. D.; Malmborg, P.; Tedroff, J.; Laangstroem, B. Appl. Radiat. Isot. 1989, 40, 171.

4. (a) Cherpillod, C.; Omer, L. M. J. Int. Med. Res. 1981, 9, 324. (b) Omer. L. M. Int J. Clin. Pharmacol. Ther. Toxicol. 1982, 20, 324.

5. (a) Brossi, A.; Teitel, S. Tetrahedron Lett. 1970, 417. (b) Schwartz, M. A.; Scott, S. W. J. Org. Chem. 1971, 36, 1827. (c) Kametani, T.; Takahashi, K.; Van Loc, C. Tetrahedron 1975, 31, 235. (d) Hart, D. J.; Cain, P. A.; Evans, D. A. J. Am. Chem. Soc. 1978, 100, 1548. (e) Irie, H.; Shiina, A.; Fushimi, T.; Katakawa, J.; Fujii, N.; Yajima, H. Chem. Lett. 1980, 875. (f) Kessar, S. V.; Singh, P.; Chawla, R.; Kumar, P. J. Chem. Soc., Chem. Commun. 1981, 1074. (g) Kametani, T.; Higashiyama, K.; Honda, T.; Otomasu, H. J. Chem. Soc., Perkin Trans. 1 1982, 2935. (h) Hara, H.; Shirai, R.; Hoshino, O.; Umezawa, B. Heterocycles 1983, $20,1945$. (i) Hara, H.; Shirai, R.; Hoshino, O.; Umezawa, B. Chem. Pharm. Bull. 1985, 33, 3107. (j) Takano, S.; Akiyama, M.; Ogasawara, K. Chem. Lett. 1985, 505. (k) Kobayashi, S.; Tokumoto, T.; Iguchi, S.; Kihara, M.; Imakura, Y.; Taira, Z. J. Chem. Res. (S) 1986, 280. (1) Narashimhan, N. S.; Patil, P. A. J. Chem. Soc., Chem. Commun. 1987, 191. (m) Gore, V. G.; 
Narashimhan, N. S. J. Chem. Soc., Perkin Trans. 1 1988, 481. (n) Katakawa, J.; Yoshimatsu, H.; Yoshida, M.; Zhang, Y.; Irie, H.; Yajima, H. Chem. Pharm. Bull. 1988, 36, 3928. (o) Couture, A.; Deniau, E.; Lebrun, S.; Grandclaudon, P. J. Chem. Soc., Perkin Trans. 1 1999, 789. (p) Toda, J.; Sonobe, A.; Ichikawa, T.; Saitoh, T.; Horiguchi, Y.; Sano, T. Arkivoc 2000, (ii), 165. (q) Honda, T.; Namiki, H.; Satoh, F. Org. Lett. 2001, 3, 631. (r) Couture, A.; Deniau, E.; Lebrun, S.; Grandclaudon, P. Tetrahedron: Asymmetry 2003, 14, 1309. (s) Couture, A.; Deniau, E.; Lebrun, S.; Grandclaudon, P. Org. Biomol. Chem. 2003, 1, 1701.

6. Parvatkar, P. T.; Parameswaran, P. S.; Tilve, S. G. Tetrahedron Lett. 2007, 48, 7870.

7. Scriven, E. F. V.; Turnbull, K. Chem. Rev. 1988, 88, 297. 